\title{
Diversity and Dynamics of Sand Flies (Diptera: Psychodidae) of Two Cutaneous Leishmaniasis Foci in the Fes-Boulemane Region of Northern Morocco
}

\author{
Fatima Zahra Talbi, ${ }^{1,2,3}$ Chafika Faraj, ${ }^{3}$ Fouad EL-Akhal, ${ }^{1}$ Fatiha El Khayyat, ${ }^{4}$ \\ Driss Chenfour, ${ }^{1}$ Abdellatif Janati Idrissi, ${ }^{1}$ and Abdelhakim El Ouali Lalami ${ }^{2,5}$ \\ ${ }^{1}$ Faculty of Sciences Dhar El Mahraz, Laboratory Biotechnology and Preservation of Natural Resources, \\ Sidi Mohamed Ben Abdellah University, 30000 Fez, Morocco \\ ${ }^{2}$ Regional Diagnostic Laboratory of Epidemiological and Environmental Health, Regional Health Directorate, \\ El Ghassani Hospital, $30000 \mathrm{Fez}$, Morocco \\ ${ }^{3}$ Laboratory of Medical Entomology, National Institute of Hygiene, 27 Avenue Ibn Battuta, Agdal, 11400 Rabat, Morocco \\ ${ }^{4}$ Laboratory of Environment and Renewable Energies, Faculty of Science, University of Ibn Tofail, 14000 Kenitra, Morocco \\ ${ }^{5}$ Institute of Nursing Professions and Health Techniques, El Ghassani Hospital, 30000 Fez, Morocco \\ Correspondence should be addressed to Abdelhakim El Ouali Lalami; eloualilalami@yahoo.fr
}

Received 29 July 2015; Revised 8 November 2015; Accepted 3 December 2015

Academic Editor: Thomas Iliffe

Copyright ( 2015 Fatima Zahra Talbi et al. This is an open access article distributed under the Creative Commons Attribution License, which permits unrestricted use, distribution, and reproduction in any medium, provided the original work is properly cited.

Cutaneous leishmaniasis (CL) is an infectious disease caused by various species of Leishmania and transmitted by several species of sand flies (Diptera: Psychodidae). In order to evaluate the risk of leishmaniasis transmission in Fes-Boulemane, an investigation was carried out in two localities, Aichoun and Bouasseme, during 2011. From January to December, 1120 specimens were collected in Aichoun comprising six species belonging to two genera: Phlebotomus sergenti (76.07\%), Phlebotomus longicuspis (9.01\%), Phlebotomus perniciosus (8.48\%), Phlebotomus papatasi (4.82\%), Sergentomyia minuta, and Sergentomyia fallax. For Bouasseme, seven species were identified with Phlebotomus sergenti (60.39\%) dominating, followed by Phlebotomus perniciosus (20\%) and Phlebotomus longicuspis (12.15\%). The remaining species, Phlebotomus papatasi, Phlebotomus ariasi, Sergentomyia minuta, and Sergentomyia fallax, were less prevalent. The activity of sand flies in both localities is marked by the dominance of Ph. sergenti with two peaks occurring in June and September. In order to obtain a better understanding of sand fly diversity among their species, results were analyzed by the ecological indices determinant: specific richness, the relative abundance, and Shannon-Weiner index $\left(H^{\prime}\right)$. Further studies of sand fly diversity should employ statistical tests and molecular analyses. This study can be useful in the implementation of appropriate future control measures.

\section{Introduction}

Cutaneous leishmaniases (CL) are parasitic diseases that remain today as a serious public health problem throughout the world. The parasitosis is caused by a "Protist Euglenobionte" that belongs to the Trypanosomatidae family, genus Leishmania. Three forms are observed: anthroponotic leishmaniasis caused by Leishmania tropica and zoonotic leishmaniasis due to L. major and L. infantum $[1,2]$. L. tropica is the most widespread and most frequently encountered. It is transmitted by Phlebotomus sergenti [3].
In Morocco, there is an annual emergence of new foci of leishmaniasis. Indeed, in 2011 and 2012, the Ministry of Health recorded, respectively, 4426 and 2990 cases with $4.92 \%$ and $7.19 \%$ in the region of Fes-Boulemane $[4,5]$. The first LC focus recorded from the Fes-Boulemane region was in 2001 with 1,600 cases only in the province of Moulay Yacoub $[6,7]$.

The objectives of our study were to identify and to make an inventory of the principle sand-flies and also to compare the specific composition and population dynamics of two biotopes located on the western and eastern slopes of the 

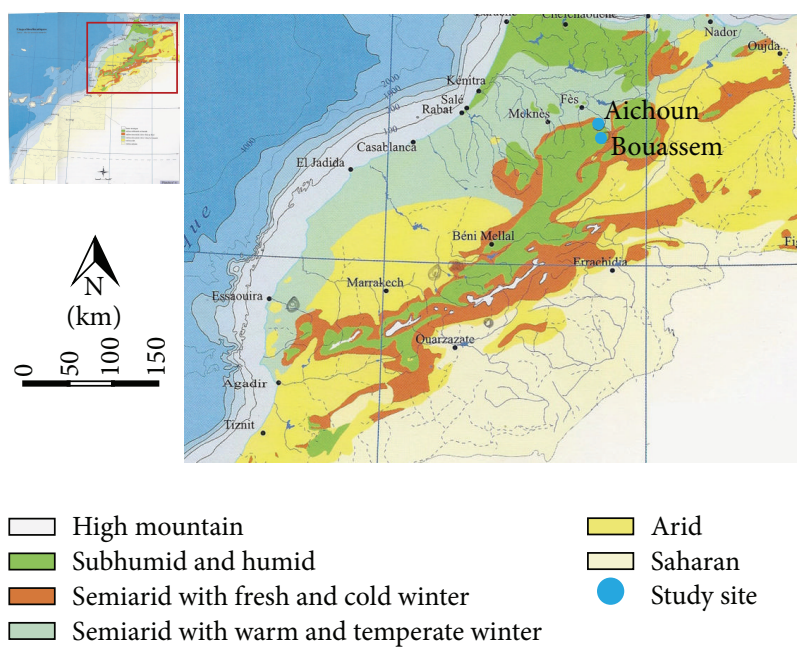

FIgURE 1: Map showing the two study site locations.

Middle Atlas, correspondingly at Aichoun and Bouasseme (Figure 1). All these steps are necessary for the surveillance and treatment of existing leishmaniases in these two foci of Fes-Boulemane region and thus contribute to its control.

\section{Materials and Methods}

2.1. Study Region. Our study area covers two ecologically and epidemiologically different localities. Aichoun $\left(33^{\circ} 39^{\prime} \mathrm{N}, 04^{\circ}\right.$ $\left.38^{\prime} \mathrm{W}\right)$ is located in the Northwest of the Middle Atlas; it is part of the province of Sefrou, belonging to the pastoral village territory of Tazouta with a cold semiarid climate. The average annual precipitation is $400 \mathrm{~mm}$, while the temperature of the coldest month is $2^{\circ} \mathrm{C}$ and the maximum of the hottest month is $40^{\circ} \mathrm{C}$.

From an epidemiological standpoint, 1242 cases of LC in the province of Sefrou were reported between 1997 and 2011 [8]. The majority of cases are due to L. tropica registered in the municipalities of El Menzel, Sefrou, Aghbalou, Tazouta Sidi Lahcen, and Ain Cheggag [9].

Bouasseme $\left(33^{\circ} 31^{\prime} \mathrm{N}, 04^{\circ} 33^{\prime} \mathrm{W}\right)$ is located in the Northeast of the Middle Atlas. It is characterized by a subhumid climate receiving about 700 to $800 \mathrm{~mm}$ of rainfall from October to May. This town is in the province of Boulemane where the first endemic cases of leishmaniasis in L. major were reported [10] (Figure 1).

2.2. Collection of Sand Flies. Sand flies were captured once every two weeks from January to December using sticky paper traps [11]. Five stations were randomly chosen. In each station, ten traps were deposited in different biotopes (stables, barns, chicken and sheep pens, etc.) and recovered the next day. The captured sand flies are kept in airtight plastic tubes containing $70 \%$ ethyl alcohol.

The females were identified based on the examination of spermathecae, cibarium, and posterior pharynx. Male identification was mainly based on examination of the genital armature. The identification was done by using morphological identification keys $[8,12]$. The morphological differentiation between the two sympatric species $P h$. longicuspis and Ph. perniciosus was made according to Berchi et al. (2007) [13]. The identification of all sand flies species was confirmed at the Laboratory of Medical Entomology in National Institute of Hygiene (the national reference laboratory in Medical Entomology). [14]:

Results are analyzed by the following ecological indices

(i) Specific richness is the number of species that make up the stand.

(ii) The relative abundance is the relative number of individuals of each species from all collected sand flies.

(iii) Shannon-Weiner index $\left(H^{\prime}\right)$ is follows:

$$
H^{\prime}=-\sum_{i=1}^{s} p_{i} \log p_{i}
$$

where " $p_{i}$ " can be calculated by dividing $n_{i}$, the number of species, by $N$, the total number of collected individuals.

\section{Results}

3.1. Specific Composition. The sand flies' activity period runs from April to October-November at the two sites. Catches by adhesive traps allowed harvesting 1120 sand flies of six species in Aichoun and 255 specimens belonging to seven species in Bouasseme. Five species belonged to the Phlebotomus genus while the other two were from the Sergentomyia genus.

These species are $P h$. sergenti which is widely distributed in the two foci, Ph. perniciosus, Ph. longicuspis, Ph. papatasi, Sergentomyia minuta, and Sergentomyia fallax. These species are common in both localities except for $P h$. ariasi which was collected only at Bouasseme (Figures 2 and 3). These species are considered the most abundant among the 23 species recorded in Morocco [8].

The specific richness, abundance, density, and ShannonWeiner index $\left(H^{\prime}\right)$ of captured sand flies are shown in Table 1. At Aichoun, the sand flies were presented with a sex ratio of 5.92. Ph. sergenti (Parrot 1917) was most abundant with 852 specimens (76.07\%). Next was Ph. longicuspis (Nitzulescu 1930) with 101 specimens (9.01\%), followed by Ph. perniciosus (Newstead 1911) with 95 specimens (8.48\%). Ph. papatasi (Scopoli 1786) was represented by $4.82 \%$. As for the other genus, Sergentomyia minuta (Rondani 1843) and Sergentomyia fallax (Parrot, 1921) were present in low relative abundance.

At Bouasseme, 255 sand flies were captured with species of Phlebotomus genus predominating both in the number of specimens captured and in their diversity (3 subgenera, 5 species). Only two species of the genus Sergentomyia were captured (Sergentomyia minuta and Sergentomyia fallax).

There was a difference in the diversity of the sand fly fauna between Aichoun and Bouasseme, as indicated by the values of Shannon-Weiner index $\left(H^{\prime}\right)$. Both localities have a value of $H^{\prime}$ less than 1, with a difference of 0.1. We compared the Shannon diversity index of all the species in each study area. 
TABLE 1: Percentage and the Shannon-Weiner diversity index $(H)$ of the sand flies collected during the study period at both localities of the Fes-Boulemane region.

\begin{tabular}{|c|c|c|c|c|c|c|c|c|}
\hline \multirow{3}{*}{ Genus } & \multirow{3}{*}{ Subgenus } & \multirow{3}{*}{ Species } & \multicolumn{6}{|c|}{ Locality (altitude m) } \\
\hline & & & \multicolumn{3}{|c|}{ Aichoun locality $(750 \mathrm{~m})$} & \multicolumn{3}{|c|}{ Bouasseme locality $(1100 \mathrm{~m})$} \\
\hline & & & $\begin{array}{c}\text { R. Ab. }{ }^{*} \\
(\%)\end{array}$ & $\begin{array}{c}\text { Shannon-Weiner } \\
\text { index }\left(H^{\prime}\right)\end{array}$ & Sex ratio & $\begin{array}{l}\text { R. Ab. }{ }^{*} \\
(\%)\end{array}$ & $\begin{array}{c}\text { Shannon-Weiner } \\
\text { index }\left(H^{\prime}\right)\end{array}$ & Sex ratio \\
\hline \multirow{5}{*}{ Phlebotomus } & & Ph. longicuspis & 9.01 & 0,11 & \multirow{5}{*}{5.9} & 12.15 & 0.09 & \multirow{6}{*}{4.7} \\
\hline & Larroussius & Ph. perniciosus & 8.48 & 0.13 & & 20 & 0.05 & \\
\hline & & Ph. ariasi & 0 & 0.02 & & 1.56 & 0 & \\
\hline & Paraphlebotomus & Ph. sergenti & 76.07 & 0.13 & & 60.39 & 0.09 & \\
\hline & Phlebotomus & Ph. papatasi & 4.82 & 0.03 & & 2.74 & 0.08 & \\
\hline Sergentomyia & & $\begin{array}{l}\text { S. minuta } \\
\text { S. fallax }\end{array}$ & $\begin{array}{c}0.89 \\
0.7\end{array}$ & $\begin{array}{l}0.02 \\
0.02\end{array}$ & & $\begin{array}{c}1.56 \\
1.5\end{array}$ & $\begin{array}{l}0.01 \\
0.01\end{array}$ & \\
\hline
\end{tabular}

${ }^{*} \mathrm{R}$. Ab.: relative abundance.

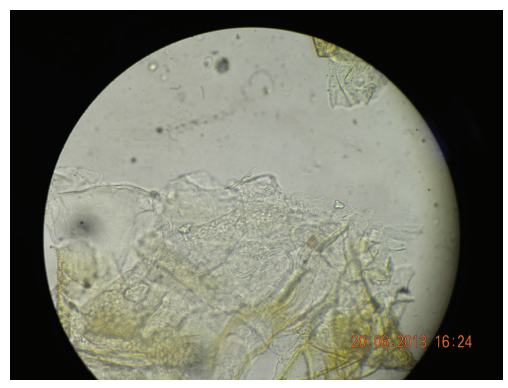

(a)

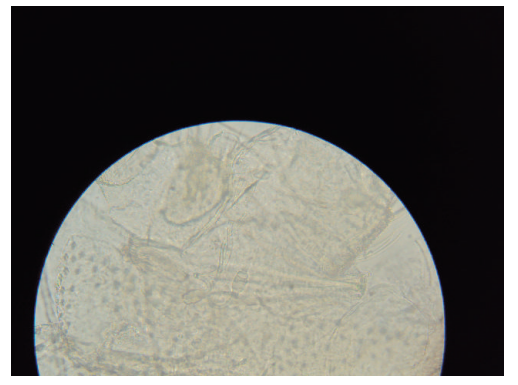

(b)

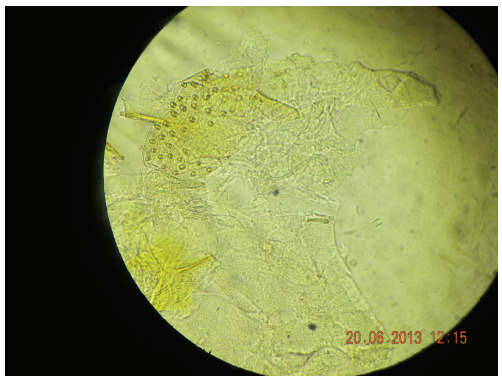

(c)

Figure 2: Female sand flies after dissection. ((a), (b)) Female genitalia spermathecal Phlebotomus sergenti; (c) Spermathecal Phlebotomus papatasi (personal photography; $G \times 40$ ).

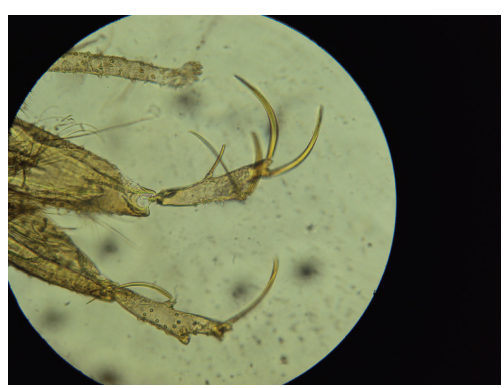

(a)

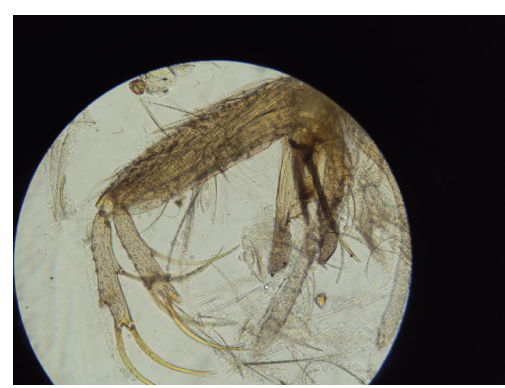

(b)

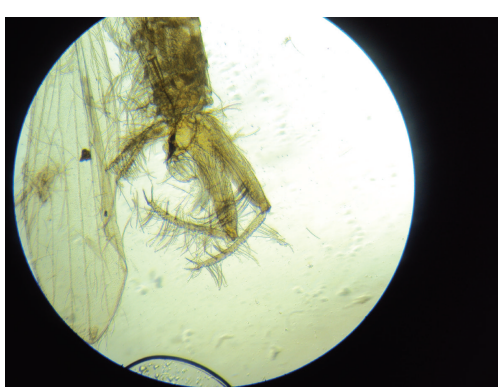

(c)

Figure 3: (a) Male genitalia and the extremity of penile valves in Phlebotomus, subgenus Larroussius; (b) male genitalia and the extremity of penile valves in Phlebotomus perniciosus; (c) male genitalia and the extremity of penile valves in Phlebotomus papatasi (personal photography; $G \times 40)$.

The values of $H^{\prime}$ at Aichoun range from 0.02 to 0.13 . The most significant value was reported in $\mathrm{Ph}$. sergenti and $\mathrm{Ph}$. longicuspis with 0.13. But the value of this index in Bouasseme for all species varies only from 0.01 to 0.09 . This difference explains why the population is more diversified at Aichoun with the predominance of $P h$. sergenti and Ph. longicuspis in comparison to Bouasseme.

3.2. Population Dynamics of Sand Flies at Aichoun and Bouasseme. The study of the activity of sand flies in these two areas was followed from January to December 2011. According to the capture sessions conducted in Aichoun and Bouasseme, we found a monthly evolution that varies from one species to another, biphasic in some species and monophasic in others. Throughout the study period, the activity of sand flies was marked by the dominance of Ph. sergenti with two peaks in June and September in both localities. In Aichoun, the activity of sand flies starts in April, coming after a gradual development observed for both $P h$. sergenti and Ph. longicuspis. After June, the sand flies' activity experienced 


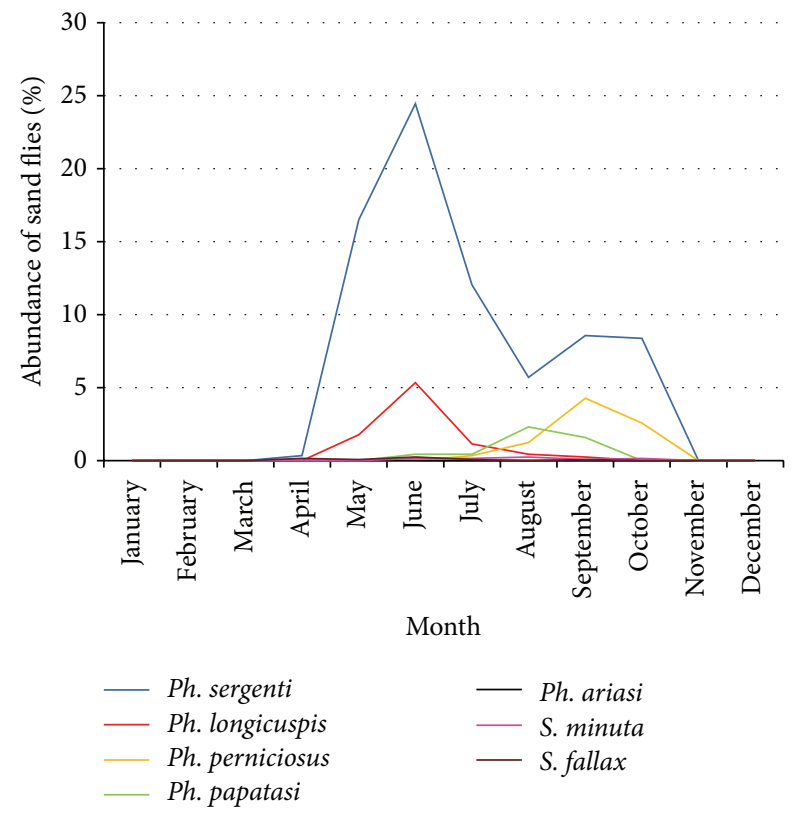

FIGURE 4: Seasonal distribution of sand flies captured at Aichoun by sticky paper traps from January to December 2011.

a fall followed by a subsequent peak for Ph. papatasi and then fell during the following months giving rise to an emergence of $P h$. perniciosus with a high percentage of $4.28 \%$ in September (Figure 4). The activity of sand flies at the Aichoun locality is spread over a period of eight months stopping in November.

At Bouasseme, sand flies appeared from May to October. Compared to Aichoun, this focus has known another representation of the seasonal fluctuation of collected species. Indeed, during the period of collections, the three species Ph. sergenti, Ph. perniciosus, and Ph. longicuspis experienced a consistent, biphasic course with peaks, June and September, as follows: Ph sergenti (26.66\%, 5.88\%), Ph. longicuspis, and Ph. perniciosus.

For Ph. ariasi, S. minuta, and S. fallax, the period of their activity was spread from June to September but with only small percentage variation (Figure 5).

\section{Discussion}

This research is a comparative study of two leishmaniasis foci in the Fes-Boulemane region of Northern Morocco in terms of abundance, species diversity, and seasonality of sand flies. During this year long entomological survey in 2011, we collected 1,120 sand flies in Aichoun and 255 in Bouasseme, two localities of the Fes-Boulemane region. The identified species belong to two genera: Phlebotomus and Sergentomyia. Species in Phlebotomus genus belong to the following three subgenera: Phlebotomus, Paraphlebotomus, and Larroussius.

In the subgenus Larroussius, we reported three species: $P h$. longicuspis, Ph. perniciosus, and Ph. ariasi. In subgenus Paraphlebotomus, we identified one species: Ph. sergenti. Ph.

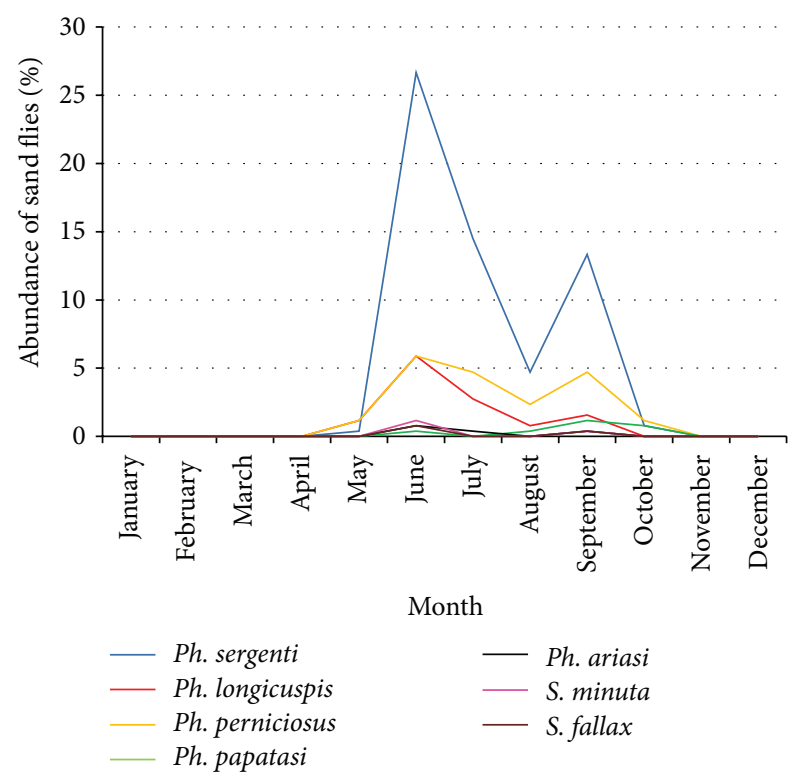

FIGURE 5: Seasonal distribution of sand flies captured at Bouasseme by adhesive traps in the period from January to December 2011.

papatasi was the sole species belonging to subgenus Phlebotomus in our collection. The two species from Sergentomyia ( $S$. minuta, $S$. fallax) were poorly represented in sand fly fauna caught in this study.

Indeed, there is a dependency between the development of leishmaniasis and density of sand flies [15].

All proven and suspected vectors of leishmaniasis in Morocco are present in the study area: Ph. sergenti is confirmed as a vector of L. tropica anthroponotic leishmaniasis in Northern Africa, the Middle East, and Central Asia [16, 17], as well as in Morocco [18]. This species, which was also suspected to be responsible for CL in Chichaoua [19], is dominant over the other species, widely dispersed in both localities and present from May to November, with peaks of activity in June and September. This vector prefers a semiarid bioclimate, even though it was captured in all bioclimatic zones and was widespread throughout Morocco [20].

These results are in agreement with those obtained by Guessouss-Idrissi et al. [21] in the province of Taza, and El Miri et al. [22] in the province of Sidi Kacem in northern Morocco. Ph. perniciosus is one of the most competent vectors of L. infantum in Mediterranean foci [23], responsible for visceral leishmaniasis in the Northeast slope of the Rif mountains where dogs are the main reservoir of the parasite [24].

In Bouasseme, seven species were trapped. They come from two genera: Phlebotomus and Sergentomyia with Ph. sergenti being dominant. Ph. ariasi and Ph. perniciosus, the usual two vectors of L. infantum in the North of the Mediterranean [25], are also represented here. The taxonomic inventory and biodiversity sand flies obtained in Aichoun during the study period in 2011 were also obtained in 2012 [26]. This confirms continuity in the sand fly development cycle.

Similar work was carried out in the Fes-Boulemane region in a focus for cutaneous leishmaniasis at Ouled Aid in 
the province of Moulay Yakoub in 2011. This study showed the same period of sand fly activity between April and November with the presence of the same species, but with different abundance [27].

From an epidemiological point of view, considering the vector-parasite relationship, we can consider Ph. sergenti as a potentially causative agent of $L$. tropica transmission in both Aichoun and Bouasseme bearing in mind that it is the only potential vector species of $L$. tropica in the study area. Given the low seasonality of $P h$. ariasi and the proven role of Ph. perniciosus and Ph. longicuspis in L. infantum [25, 28], these two species are likely the vectors of $L$. infantum in the study area. Ph. papatasi, the proved vector of $L$. major in Morocco [29], has a low density at both sites; this could be explained by its preference for an arid climate [30]. The high abundance of the sand flies species in Aichoun and Bouasseme can be explained by environmental conditions, especially temperature and humidity which are conducive to a rapid biological development.

\section{Conclusion and Perspectives}

The wide distribution of sand flies and the long activity period of Ph. sergenti, Ph. longicuspis, and Ph. perniciosus in both foci Aichoun and Bouasseme indicate that the risk of transmission of leishmaniasis is potentially higher in this region. These results will serve as a database for further work in these two localities. They suggest the necessity for continuous entomological surveillance to lessen the risk of transmission of cutaneous leishmaniasis.

In perspective, we propose that further studies of sand fly diversity be conducted through molecular analyses, in order to positively identify sand fly species.

The determination of climatic factors favorable for sand flies development and the study of host-blood feeding preferences are also useful for developing a control program, directing operations, and evaluating their effectiveness.

\section{Conflict of Interests}

The authors declare that they have no conflict of interests.

\section{Acknowledgments}

The authors thank the Medical Entomology Laboratory team at the National Institute of Hygiene of Rabat, for their constant support provided during this study. Likewise, the authors thank the regional direction of the Fes-Boulemane Health, Delegate of Ministry of Health, in Sefrou province, and staff at Aichoun and Bouasseme for their cooperation, assistance, and information.

\section{References}

[1] M. Mohebali, E. Javadian, M. R. Yaghoobi-Ershadi, A. A. Akhavan, H. Hajjaran, and M. R. Abaei, "Characterization of Leishmania infection in rodents from endemic areas of the Islamic republic of Iran," Eastern Mediterranean Health Journal, vol. 10, no. 4-5, pp. 591-599, 2004.
[2] M. R. Yaghoobi-Ershadi, A. A. Hanafi-Bojd, E. Javadian, R. Jafari, A. R. Zahraei-Ramazani, and M. Mohebali, "A new focus of cutaneous leishmaniasis caused by Leishmania tropica," Saudi Medical Journal, vol. 23, no. 3, pp. 291-294, 2002.

[3] E. Guilvard, J. A. Rioux, M. Gallego et al., "Leishmania tropica in Morocco. III-the vector of Phlebotomus sergenti. Apropos of 89 isolates," Annales de Parasitologie Humaine et Comparee, vol. 66, no. 3, pp. 96-99, 1991.

[4] Ministry of Health and DELM, Department of Planning and Financial Resources, Directorate of Planning and Research Services Research and Health Information, Health Digit Edit, 2012.

[5] Ministry of Health and DELM, Department of Planning and Financial Resources, Directorate of Planning and Research Services Research and Health Information, Health Digit Edit, 2013.

[6] H. Fellah, M. Rhajaoui, S. Ouahabi, D. Belghiti, and M. Lyagoubi, "Occurrence of human cutanous leishmanioasis in zouagha my yacoub province (Morocco)," International Journal of Agriculture and Biology, vol. 9, no. 1, pp. 197-198, 2007.

[7] M. Rhajaoui, "Human leishmaniases in Morocco: a nosogeographical diversity," Pathologie Biologie, vol. 59, no. 4, pp. 226229, 2011.

[8] Ministry of Health, Fight against Leishmaniasis, Activity Guide, Ministry of Health, Rabat, Morocco, 2010.

[9] H. Asmae, A. Fatima, F. Hajiba et al., "Coexistence of Leishmania tropica and Leishmania infantum in Sefrou province, Morocco," Acta Tropica, vol. 130, no. 1, pp. 94-99, 2014.

[10] M. Rhajaoui, N. Abedelmajeed, H. Fellah et al., "Molecular typing reveals emergence of a new clinic-epidemiologic profile of cutaneous leishmanisis in Morocco," Emerging Infectious Diseases journal, vol. 3, no. 9, pp. 1358-1360, 2007.

[11] M. M. Emami and M. Yazdi, "Entomological survey of phlebotomine sand flies (Diptera: Psychodidae) in a focus of visceral leishmaniasis in central Iran," Journal of Vector Borne Diseases, vol. 45, no. 1, pp. 38-43, 2008.

[12] B. Pesson, J. S. Ready, I. Benabdennbi et al., "Sandflies of the Phlebotomus perniciosus complex: mitochondrial introgression and a new sibling species of P. longicuspis in the Moroccan Rif," Medical and Veterinary Entomology, vol. 18, no. 1, pp. 25-37, 2004.

[13] S. Berchi, A. Bounamous, K. Louadi, and B. Pesson, "Morphological distinction between two sympatric species: Phlebotomus pernlciosus and Phlebotomus longicuspis (Diptera: Psychodidae)," Annales de la Société Entomologique de France, vol. 43, no. 2, pp. 201-203, 2007.

[14] N. Kabbout, D. Merzoug, and H. Chenchouni, "Ecological status of phlebotomine sand flies (Diptera: Psychodidae) in rural communities of Northeastern Algeria," Journal of ArthropodBorne Diseases, vol. 10, no. 1, pp. 24-38, 2015.

[15] L. Fernando Chaves, E. José, A. Valderrama, and A. Saldaña, "Cutaneous leishmaniasis and sand fly fluctuations are associated with el niño in panamá," PLoS Neglected Tropical Diseases, vol. 10, no. 1, pp. 1-11, 2014.

[16] K. Mondragon-Shem, W. S. Al-Salem, L. Kelly-Hope et al., "Severity of old world cutaneous leishmaniasis is influenced by previous exposure to sandfly bites in Saudi Arabia," PLoS Neglected Tropical Diseases, vol. 9, no. 2, pp. 1-14, 2015.

[17] A. Tabbabi, N. Bousslimi, A. Rhim, K. Aoun, and A. Bouratbine, "Short report: first report on natural infection of Phlebotomus sergenti with Leishmania promastigotes in the cutaneous leishmaniasis focus in southeastern Tunisia," The American Journal 
of Tropical Medicine and Hygiene, vol. 85, no. 4, pp. 646-647, 2011.

[18] F. Pratlong, J. A. Rioux, J. Dereure et al., "Leishmania tropica au Maroc IV-Diversité isozymique intrafocale," Annales de Parasitologie Humaine et Comparée, vol. 66, no. 3, pp. 100-104, 1991.

[19] S. Guernaoui, A. Boumezzough, B. Pesson, and G. Pichon, "Entomological investigations in Chichaoua: an emerging epidemic focus of cutaneous leishmaniasis in Morocco," Journal of Medical Entomology, vol. 42, no. 4, pp. 697-701, 2005.

[20] S. Boussaa, M. Neffa, B. Pesson, and A. Boumezzough, "Phlebotomine sand flies (Diptera: Psychodidae) of Southern Morocco: results of entomological surveys along the Marrakech-Ouarzazat and Marrakech-Azilal roads," Annals of Tropical Medicine and Parasitology, vol. 104, no. 2, pp. 163-170, 2010.

[21] N. Guessouss-Idrissi, B. Berrag, M. Riyad, H. Sahibi, M. Bichichi, and A. Rhalem, "Leishmania tropica: etiologie agent of a case of visceralizing canine leishmaniasis in north Morocco," The American Journal of Tropical Medicine and Hygiene, vol. 57, no. 2, pp. 172-173, 1997.

[22] H. El Miri, M. Rhajaoui, O. Himmi, S. Ouahabi, A. Benhoussa, and C. Faraj, "Entomological study of five cutaneous leishmaniasis foci in the Sidi Kacem province, north Morocco," Annales de la Société entomologique de France, vol. 49, no. 2, pp. 154-159, 2013.

[23] R. Benikhlef, Z. Harrat, M. Toudjine, A. Djerbouh, S. BendaliBraham, and M. Belkaid, "Detection of Leishmania infantum MON-24 in the dog," Medecine Tropicale: Revue du Corps de Santé Colonial, vol. 64, no. 4, pp. 381-383, 2004.

[24] R. Nejjar, M. Lemrani, A. Malki, S. Ibrahimy, H. Amarouch, and A. Benslimane, "Canine leishmaniasis due to Leishmania infantum MON-1 in northern Morocco," Parasite, vol. 5, no. 4, pp. 325-330, 1998.

[25] M. Durán-Martínez, E. Ferroglio, P. Acevedo et al., "Leishmania infantum (Trypanosomatida: Trypanosomatidae) phlebotomine sand fly vectors in continental mediterranean Spain," Environmental Entomology, vol. 42, no. 6, pp. 1157-1165, 2013.

[26] F. Z. Talbi, A. El Ouali Lalami, A. Janati Idrissi, F. Sebti, and C. Faraj, "Leishmaniasis in central Morocco: seasonal fluctuations of phlebotomine sand fly in Aichoun locality, from Sefrou province," Pathology Research International, vol. 2015, Article ID 438749, 4 pages, 2015.

[27] K. Lahouiti, A. El Ouali Lalami, S. Maniar, and K. Bekhti, "Seasonal fluctuations of phlebotomines sand fly populations (Diptera: Psychodidae) in the Moulay Yacoub province, centre Morocco: effect of ecological factors," African Journal of Environmental Science and Technology, vol. 7, no. 11, pp. 1028-1036, 2013.

[28] R. Killick-Kendrick, "Phlebotomine vectors of the leishmaniases: a review," Medical and Veterinary Entomology, vol. 4, no. 1, pp. 1-24, 1990.

[29] D. Fryauff and H. Hanafi, "Demonstration of hybridization between Phlebotomus papatasi (Scopoli) and Phlebotomus bergeroti Parrot," Parassitologia, vol. 33, pp. 237-243, 1991.

[30] S. Guernaoui, A. Boumezzough, and A. Laamrani, "Altitudinal structuring of sand flies (Diptera: Psychodidae) in the HighAtlas mountains (Morocco) and its relation to the risk of leishmaniasis transmission," Acta Tropica, vol. 97, no. 3, pp. 346$351,2006$. 

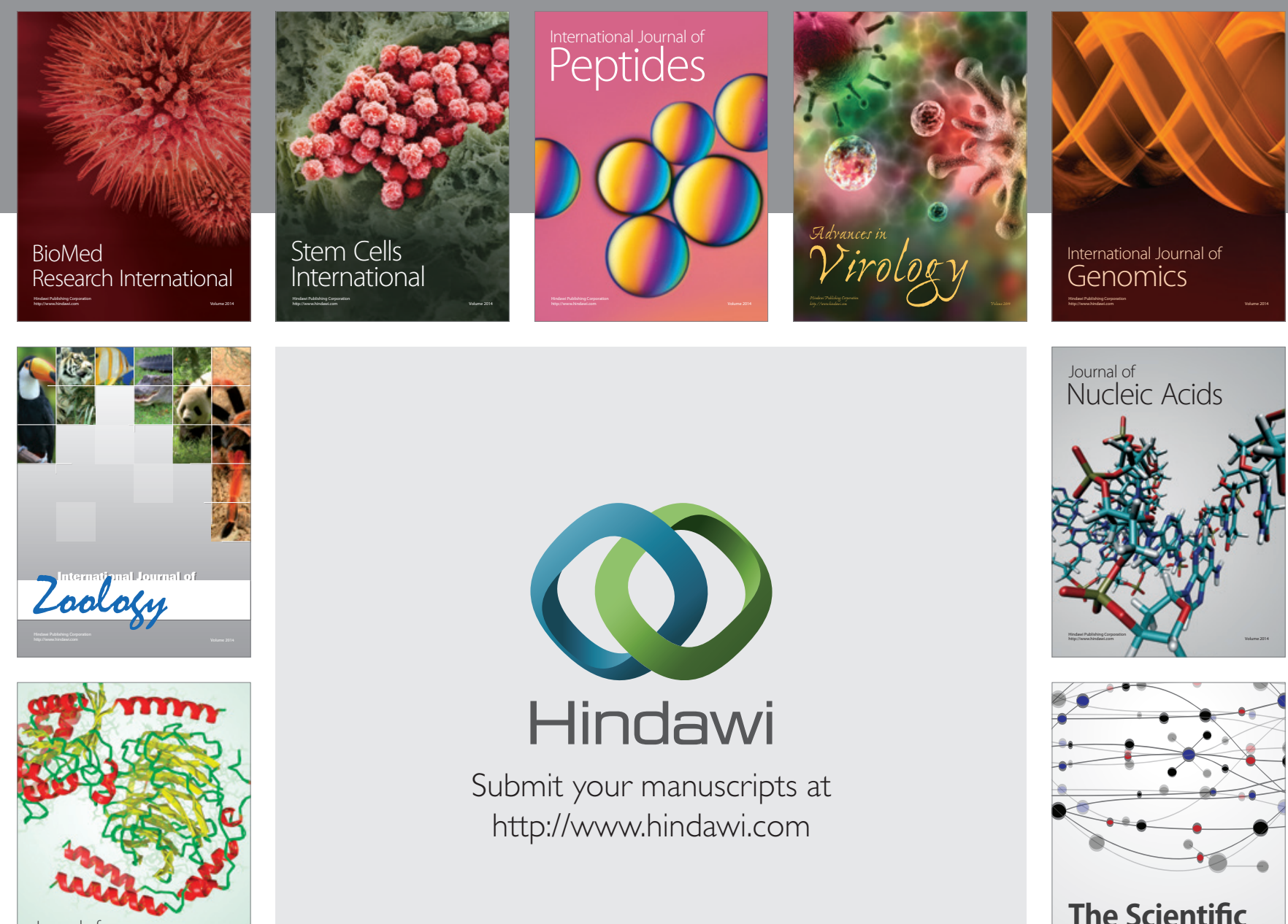

Submit your manuscripts at

http://www.hindawi.com

Journal of
Signal Transduction
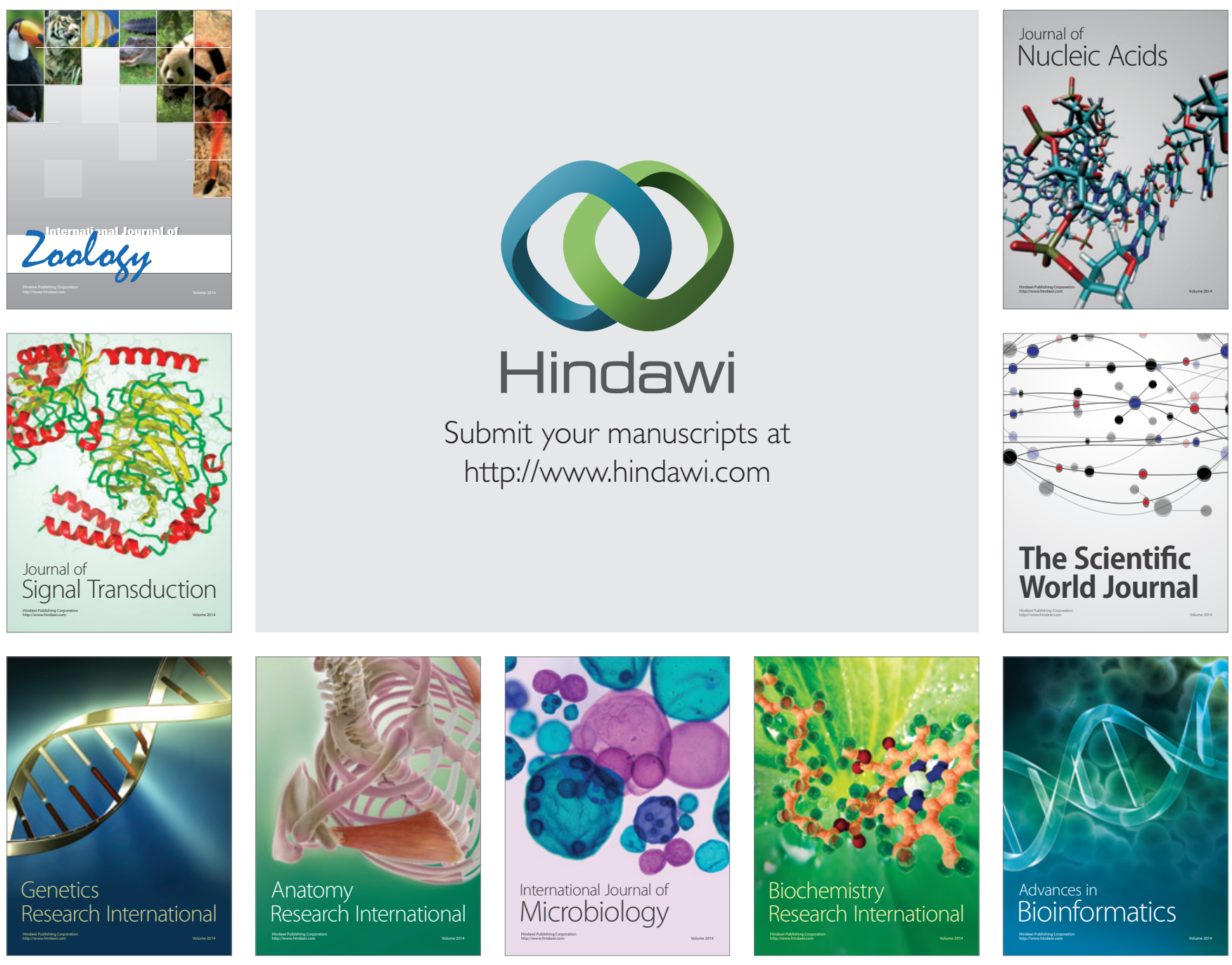

The Scientific World Journal
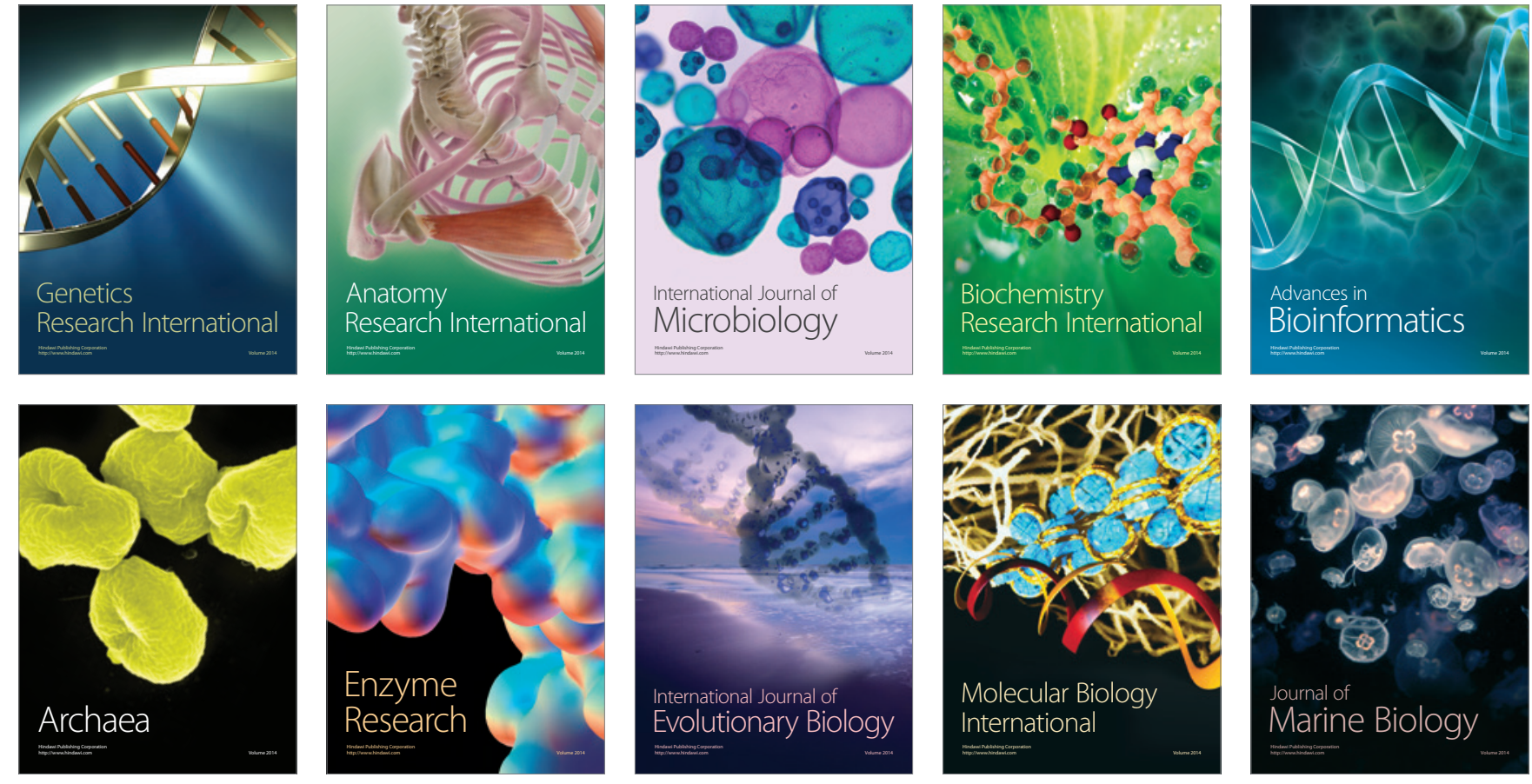